\title{
ROTATION HYPERSURFACES IN SPACES OF CONSTANT CURVATURE
}

\author{
BY
}

\author{
M. DO CARMO AND M. DAJCZER
}

\begin{abstract}
Rotation hypersurfaces in spaces of constant curvature are defined and their principal curvatures are computed. A local characterization of such hypersurfaces, with dimensions greater than two, is given in terms of principal curvatures. Some special cases of rotation hypersurfaces, with constant mean curvature, in hyperbolic space are studied. In particular, it is shown that the well-known conjugation between the belicoid and the catenoid in euclidean three-space extends naturally to hyperbolic three-space $H^{3}$; in the latter case, catenoids are of three different types and the explicit correspondence is given. It is also shown that there exists a family of simply-connected, complete, embedded, nontotally geodesic stable minimal surfaces in $H^{3}$.
\end{abstract}

1. Introduction. The main purpose of the present paper is to extend the classical notion of rotation surfaces of the three-dimensional euclidean space $R^{3}$ to hypersurfaces of the $(n+1)$-dimensional hyperbolic space $H^{n+1}(c)$ with constant curvature $c<0$. Our primary motivation was to describe explicitly some simple examples of minimal hypersurfaces of $H^{n+1}(c)$ and to study their properties, in particular, their stability properties. It soon became clear, however, that it would probably be useful to frame some of our results in a more general context.

In $\$ 2$ we define rotation hypersurfaces of $H^{n+1}(c)$ and give their explicit parametrizations. Roughly speaking, they are generated by moving an $(n-1)$-dimensional umbilic submanifold $\Sigma \subset H^{n+1}(c), \Sigma$ with varying curvature, along a certain curve (cf. Definition (2.2)). The various positions of $\Sigma$ are called the parallels of the rotation hypersurface which is then classified according to the nature of the parallels: spherical, if the parallels are spheres, hyperbolic, if the parallels are hyperbolic spaces, and parabolic, if the parallels are horospheres. It seems to us that the two last types have not appeared explicitly in the literature. Actually, we were led to them by considering the one-parameter family of geodesically-ruled minimal surfaces in $H^{3}(-1)$ (see Lawson [7, p. 352], and §3). By analogy with the situation in $R^{3}$, where helicoids are conjugate to catenoids, it is natural to expect that each such surface, say, corresponding to a parameter $\lambda \in(0, \infty)$ is conjugate (in a sense to be made precise, see §3) to a rotation minimal surface $M_{\lambda} \subset H^{3}(-1)$. This is indeed the case if we bring into play all the three types considered above and the correspondence is actually very interesting: for $\lambda \in(0,1), M_{\lambda}$ is a rotation minimal surface of spherical type, for $\lambda \in(1, \infty), M_{\lambda}$ is a rotation minimal surface of hyperbolic type, and

Received by the editors May 22, 1981 and, in revised form, May 19, 1982.

1980 Mathematics Subject Classification. Primary 53C40, 53C42, 53A10; Secondary 49F10.

(C)1983 American Mathematical Society 0002-9947/82/0000-0568/\$06.50 
for $\lambda=1, M_{\lambda}$ is the unique minimal rotation surface of parabolic type in $H^{3}(-1)$. (That it is unique is proved in a more general context in Theorem (3.14); the result itself is proved in Theorem (3.31).)

For convenience of later theorems, we formulate the definition of rotation hypersurfaces in a way that it applies equally well when the ambient space is the sphere $S^{n+1}(c)$, with constant curvature $c>0$, or the euclidean space $R^{n+1}$. From now on, we will denote by $\bar{M}^{n+1}(c)$, a simply-connected, complete, Riemannian manifold with constant curvature $c$, where $c$ is a real number.

In classical differential geometry, rotation surfaces with an additional property (constant mean curvature, constant Gaussian curvature, minimal, etc.) are probably the simplest surfaces having the specified property, and provides a good test-ground for various conjectures. Of course, similar questions can be posed for the hypersurfaces here defined. In $\S 3$ we compute the principal curvatures $\lambda$ and $\mu$ of rotation hypersurfaces; one of them, say $\lambda$, has multiplicity at least $n-1$. By specifying a certain property in the form $\theta(\lambda, \mu)=0$, a differential equation is determined that yields a family of rotation hypersurfaces with the specified property. As an example, to be used extensively in the paper, we establish the equation of the rotation hypersurfaces with prescribed mean curvature and use it to prove that up to isometries of $H^{n+1}(c)$, there exists a unique complete parabolic rotation hypersurface of $H^{n+1}(c)$ with a given constant mean curvature $H$. If $H=0$, such a hypersurface is an embedded, simply-connected, (minimal) hypersurface (Theorem (3.14)).

The question whether complete rotation minimal hypersurfaces are embedded is quite interesting. We can prove that if $f: M^{2} \rightarrow H^{3}(-1)$ is a rotation minimal surface, then $f$ is an embedding (Theorem (3.26)). From a different point of view, the embeddability of rotation minimal hypersurfaces of $S^{n+1}(c)$ has been treated by Otsuki in a long series of papers that started with [10]. We conclude $\$ 3$ by proving the conjugacy stated in the beginning of the Introduction.

In $\S 4$ we restrict ourselves to the case $n \geqslant 3$, and present some sufficient conditions for an arbitrary hypersurface $f: M^{n} \rightarrow \bar{M}^{n+1}(c)$ to be a rotation hypersurface. The main result is that if the principal curvatures $k_{1}, \ldots, k_{n}$ of $f$ satisfy $k_{1}=k_{2}=\cdots=k_{n-1}=-\lambda \neq 0, k_{n}=-\mu=-\mu(\lambda)$, and $\lambda-\mu \neq 0$, then $f(M)$ is contained in a rotation hypersurface (Theorem (4.2)). This is trivially not true if $n=2$ and generalizes a result of Otsuki [10] who proved it for $c>0$ and $f$ minimal. As a corollary, we obtain that a conformally flat hypersurface $f: M^{n} \rightarrow \bar{M}^{n+1}(c), n \geqslant 4$ (it is known that in this case $\left.k_{1}=k_{2}=\cdots=k_{n-1}=-\lambda\right)$, that admits a relation $\mu=\mu(\lambda)$, with $\lambda \neq 0$ and $\lambda-\mu \neq 0$, is contained in a rotation hypersurface. This generalizes a result of Blair [2] who proved it for $c=0$ and $f$ minimal.

A further sufficient condition is related to the determination of hypersurfaces $f$ : $M^{n} \rightarrow \bar{M}^{n+1}(c)$ that, roughly speaking, satisfy a specified condition and are invariant by an $l$-parameter group of isometries of $\bar{M}^{n+1}(c)$. The main result is that if $l$ is the maximum admissable value then either $f$ has two principal curvatures $\lambda, \mu$, where one of them has at least multiplicity $n-1$ or $f$ is contained in an isoparametric family (in the sense of E. Cartan [4]) of $M^{n+1}(c)$ (Theorem (4.7)). In the particular case that $f$ is minimal and $c \leqslant 0$, a more precise result can be obtained, namely (Corollary 
(4.9)): Let $f: M^{n} \rightarrow \bar{M}^{n+1}(c), c \leqslant 0, n \geqslant 3$, be a nontotally geodesic, minimal immersion, invariant by an l-parameter subgroup of the isometries of $\bar{M}^{n+1}(c)$. Then $l_{\max }=n(n-1) / 2$ and if $l=l_{\max }, f(M)$ is a rotation hypersurface. This generalizes a result of Barbosa and do Carmo [1], who proved it for $c=0$. The result does not hold for $c>0$.

Finally, in $\$ 5$ we study some stability properties of rotation minimal surfaces in hyperbolic spaces. H. Mori [8] has shown that a certain class of complete spherical rotation minimal surfaces in $H^{3}(-1)$ is globally stable. Here we show that the complete hyperbolic and parabolic rotation minimal surfaces are all globally stable. It follows (Theorem (5.5)) that there exists a family of simply-connected, complete, nontotally geodesic, embedded stable minimal surfaces in $H^{3}(-1)$.

Next, we derive (Theorem (5.6)) a necessary condition for a complete minimal hypersurface of $H^{n+1}(c)$ to be globally stable. The condition is an integral inequality on the norm of the second fundamental form that is obtained by a modification of the argument in do Carmo and Peng [3]. With this inequality it is possible to show that there are spherical catenoids that are not globally stable.

We want to thank $\mathrm{H}$. Mori for kindly pointing out to us a mistake in a constant appearing in our first version of Theorem (5.6) and for communicating to us the corrected estimate for the range of unstable spherical catenoids. We also want to thank Oscar Bustos at IMPA for the computer work needed in Theorem (3.26). Finally, we want to thank the referee for a number of valuable comments.

\section{General properties.}

(2.1) In this section we define the rotation hypersurfaces of $\bar{M}^{n+1}(c)$ and write down their parametrizations. We begin with the case where $c<0$, and we will first establish some notation.

We will denote by $L^{n}$ the space of $n$-tuples $\left(x_{1}, \ldots, x_{n}\right)=x$ with the Lorentzian metric $g_{-1}(x, y)=-x_{1} y_{1}+\cdots+x_{n} y_{n}$, where $y=\left(y_{1}, \ldots, y_{n}\right)$, and will consider the hyperbolic space $H^{n+1}(c), c<0$, as a hypersurface of $L^{n+2}$, namely,

$$
H^{n+1}(c)=\left\{x \in L^{n+2} ; g_{-1}(x, x)=1 / c, x_{1}>0\right\} .
$$

An orthogonal transformation of $L^{n+2}$ is a linear map that preserves the bilinear form $g_{-1}$; the orthogonal transformations induce, by restriction, all the isometries of $H^{n+1}(c)$. We will denote by $P^{k}$ a $k$-dimensional subspace of $L^{n+2}$ passing through the origin and by $O\left(P^{2}\right)$ the set of orthogonal transformations of $L^{n+2}$ with positive determinant that leave $P^{2}$ pointwise fixed. We will also use $\left[v_{1}, \ldots, v_{k}\right]$ to denote the subspace generated by the vectors $v_{1}, \ldots, v_{k}$. We will say that $P^{k}$ is Lorentzian if the restriction $g_{-1} \mid P^{k}$ is a Lorentzian metric; $P^{k}$ is Riemannian if $g_{-1} \mid P^{k}$ is a Riemannian metric; $P^{k}$ is degenerate if $g_{-1} \mid P^{k}$ is a degenerate quadratic form. Sometimes, it will be convenient to consider affine subspaces of $L^{n+2}$ rather than subspaces passing through the origin; we will distinguish them by a bar placed above the letter.

(2.2) Definition. Choose $P^{2}$ and $P^{3} \supset P^{2}$ such that $P^{3} \cap H^{n+1}(c) \neq \varnothing$. Let $C$ be a regular curve in $P^{3} \cap H^{n+1}(c)=H^{2}(c)$ that does not meet $P^{2}$. The orbit of $C$ under the action of $O\left(P^{2}\right)$ is called a rotation hypersurface $M^{n} \subset H^{n+1}(c)$ generated 
by $C$ around $P^{2}$. If $o \in O\left(P^{2}\right)$, the curve $o(C)$ is a meridian of $M^{n}$ and the orbit of a point of $C$ under $O\left(P^{2}\right)$ is a parallel of $M^{n}$.

(2.3) REMARK. A hypersurface of rotation $M^{n} \subset R^{n+1}$ generated by a curve $C$ around an axis $r$ that does not meet $C$ is obtained by taking the orbit of $C$ under those orthogonal transformations of $R^{n+1}$ that leaves $r$ pointwise fixed. By setting $R^{n+1}=\left\{\left(x_{1}, \ldots, x_{n+2}\right) \in R^{n+2} ; x_{n+2}=0\right\}$ and taking $P^{2}=r \oplus e_{n+2}, e_{n+2}=$ $(0, \ldots, 1)$, we see that $M^{n}$ satisfies Definition (2.2) with $R^{n+1}$ in place of $H^{n+1}(c)$.

(2.4) We have yet to show that $M^{n}$, defined by Definition (2.2), is actually a hypersurface of $H^{n+1}(c)$. One way of doing this is to give $M^{n}$ an explicit parametrization. For that, we need a description of $O\left(P^{2}\right)$, and we must distinguish three exhaustive cases:

(i) (The spherical case) $P^{2}$ is Lorentzian.

(ii) (The hyperbolic case) $P^{2}$ is Riemannian.

(iii) (The parabolic case) $P^{2}$ is degenerate.

In the first two cases, it is easily seen that we can choose an orthonormal basis $e_{1}, \ldots, e_{n+1}, e_{n+2}$ of $L^{n+2}$ such that the following conditions are satisfied:

(1) $P^{2}$ is the plane $\left[e_{n+1}, e_{n+2}\right]$ generated by $e_{n+1}$ and $e_{n+2}$,

(2) $g_{-1}\left(e_{n+2}, e_{n+2}\right)=-1$ in case (i), and $g_{-1}\left(e_{1}, e_{1}\right)=-1$ in case (ii),

(3) the matrix of an element of $O\left(P^{2}\right)$ can be written in this basis as

$$
\left(\begin{array}{ccccc}
A_{1} & & & & \\
& A_{2} & & & \\
& & \ddots & & \\
& & & A_{n / 2} & \\
& & & & I
\end{array} \mid \text { for } n\right. \text { even, }
$$

or

$$
\left(\begin{array}{cccccc}
A_{1} & & & & & \\
& A_{2} & & & \\
& & \ddots & & \\
& & & A_{(n-1) / 2} & & \\
& & & & 1 & \\
& & & & & I
\end{array}\right) \text { for } n \text { odd, }
$$

where $I$ is the $2 \times 2$ identity matrix, $A_{1}$ is a $2 \times 2$ matrix that has the form

$$
\begin{aligned}
R\left(\theta_{1}\right) & =\left(\begin{array}{cc}
\cos \theta_{1} & \sin \theta_{1} \\
-\sin \theta_{1} & \cos \theta_{1}
\end{array}\right) \quad \text { in case (i), } \\
& =\left(\begin{array}{cc}
\cosh \theta_{1} & \sinh \theta_{1} \\
\sinh \theta_{1} & \cosh \theta_{1}
\end{array}\right) \quad \text { in case (ii) }
\end{aligned}
$$

and, in both cases, $A_{i}=R\left(\theta_{i}\right)$ for $i>1$. 
Case (iii) is less usual. To treat it, we consider a pseudo-orthonormal basis of $L^{n+2}$, i.e., a basis $e_{1}, \ldots, e_{n+2}$ such that

$$
\begin{gathered}
g_{-1}\left(e_{1}, e_{1}\right)=g_{-1}\left(e_{n+1}, e_{n+1}\right)=0, \quad g_{-1}\left(e_{1}, e_{n+1}\right)=1, \\
g_{-1}\left(e_{k}, e_{j}\right)=\delta_{k j}, \quad k=1, \ldots, n+2, j=2, \ldots, n+2, j \neq n+1 .
\end{gathered}
$$

In such a basis,

$$
g_{-1}(x, x)=2 x_{1} x_{n+1}+x_{2}^{2}+\cdots+x_{n}^{2}+x_{n+2}^{2}, \quad x=\sum x_{k} e_{k} .
$$

Assume that the pseudo-orthonormal basis is so chosen that $P^{2}=\left[e_{n+1}, e_{n+2}\right]$. Consider the $(n+2) \times(n+2)$ matrices given by

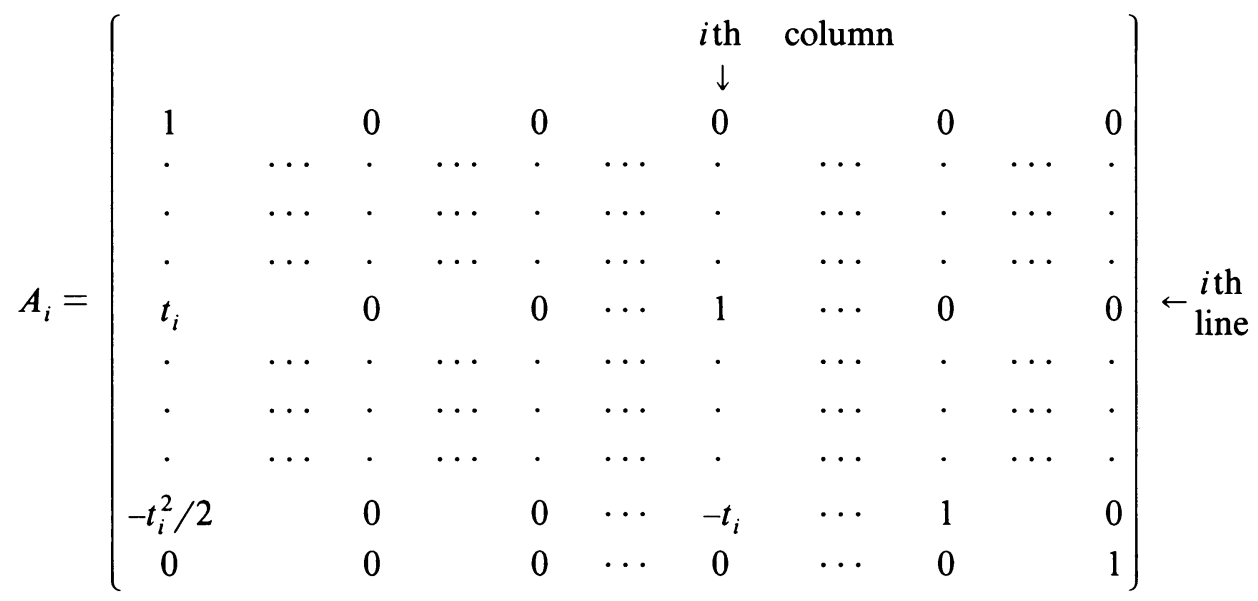

where $2 \leqslant i \leqslant n$, and the $(n-2)$ nonwritten lines make up a matrix of the form

$$
\left[\begin{array}{cccccccccc}
0 & b_{22}^{i} & b_{23}^{i} & \cdots & \overbrace{0}^{i} & b_{2, i+1}^{i} & \cdots & b_{2 n}^{i} & 0 & 0 \\
\vdots & b_{32}^{i} & b_{33}^{i} & \cdots & 0 & b_{3, i+1}^{i} & \cdots & b_{3 n}^{i} & 0 & 0 \\
\vdots & \vdots & \vdots & & \vdots & \vdots & & \vdots & \vdots & \vdots \\
0 & b_{n 2}^{i} & b_{n 3}^{i} & \cdots & 0 & b_{n, i+1}^{i} & \cdots & b_{n n}^{i} & 0 & 0
\end{array}\right) \text {, }
$$

where $\left(b_{\alpha \beta}^{i}\right)=B_{i}, \alpha, \beta=2, \ldots, n, \alpha \neq i, \beta \neq i$, is an $(n-2) \times(n-2)$ orthogonal matrix. We claim that $A_{i}$ are matrices of elements of $O\left(P^{2}\right)$ in the above basis and that they are all of them.

Clearly $A_{i}$ leaves $P^{2}$ pointwise fixed. To show that $A_{i}$ preserves $g_{-1}$, we notice that if $x=\sum x_{k} e_{k}, k=1, \ldots, n+2$, then

$$
A_{i} x=\left(x_{1}, \bar{x}_{2}, \bar{x}_{3}, \ldots, t_{i} x_{1}+x_{i}, \ldots,-\frac{t_{i}^{2}}{2} x_{1}-t_{i} x_{i}+x_{n+1}, x_{n+2}\right),
$$

where $\bar{x}_{\alpha}$ is defined by

$$
B_{i}\left(\sum_{\alpha} x_{\alpha} e_{\alpha}\right)=\left(\bar{x}_{2}, \bar{x}_{3}, \ldots, \bar{x}_{n}\right), \text { i.e., } \sum x_{\alpha}^{2}=\sum \bar{x}_{\alpha}^{2} .
$$


Thus

$$
\begin{aligned}
g_{-1}\left(A_{i} x, A_{i} x\right) & =2 x_{1}\left(-\frac{t_{i}^{2}}{2} x_{1}-t_{i} x_{i}+x_{n+1}\right)+\left(t_{i} x_{1}+x_{i}\right)^{2}+x_{n+2}^{2}+\sum_{\alpha} \bar{x}_{\alpha}^{2} \\
& =2 x_{1} x_{n+1}+x_{i}^{2}+x_{n+2}^{2}+\sum_{\alpha} x_{\alpha}^{2}=g_{-1}(x, x),
\end{aligned}
$$

and this proves the first part of our claim.

To show that we have obtained in this way all the elements of $O\left(P^{2}\right)$, we make the following observation. The intersection of $H^{n+1}(c)$ with an affine $n$-plane of $L^{n+2}$ parallel to $\left[e_{1}, \ldots, e_{n}\right]$ is a "paraboloid" of $L^{n+2}$ that represents a $(n-1)$-horosphere of $H^{n+1}$. Clearly $O\left(P^{2}\right)$ is the group of isometries of such a horosphere; hence the dimension of $O\left(P^{2}\right)$ is $n(n-1) / 2$. On the other hand, when $B_{i}=$ ident., the matrix $A_{i}$ is that isometry of the paraboloid that takes into itself the parabola contained in a plane parallel to $\left[e_{1}, e_{i}\right]$. Furthermore, each $B_{i}$ is an isometry of the sphere in which the paraboloid is cut by subspaces parallel to $\left[e_{2}, e_{3}, \ldots, \hat{e}_{i}, \ldots, e_{n}\right]$, where $\hat{e}_{i}$ means that $e_{i}$ is omitted. By varying $i$ from 2 to $n$, we see that the dimension of the set of $B_{i}$ 's is the dimension of the group of isometries of the sphere in which the paraboloid is cut by a subspace parallel to $\left[e_{2}, e_{3}, \ldots, e_{i}, \ldots, e_{n}\right]$, namely $(n-2)(n-1) / 2$. Thus the dimension of the matrices of type $A_{i}$ is

$$
(n-1)+(n-2)(n-1) / 2=n(n-1) / 2,
$$

which is the dimension of $O\left(P^{2}\right)$ and proves our claim.

(2.5) We are now ready to parametrize the orbit of $C$ in Definition (2.2). If $P^{2}$ is in case (i) or (ii) above, choose an orthonormal basis of $L^{n+2}$ such that conditions (1), (2) and (3) of (2.4) hold. If $P^{2}$ is case (iii), choose a pseudo-orthonormal basis as above. In both cases, let the space $P^{3} \supset P^{2}$ in Definition (2.2) be given by $P^{3}=\left[e_{1}, e_{n+1}, e_{n+2}\right]$ and parametrize the curve $C$ in $P^{3} \cap H^{n+1}$ by $x_{1}=x_{1}(s)$, $x_{n+1}=x_{n+1}(s), x_{n+2}=x_{n+2}(s)$.

Now, for a fixed $s=s_{0}$, the intersection $U\left(s_{0}\right)$ of $H^{n+1}$ with an affine $n$-plane passing through $\left(0,0, \ldots, 0, x_{n+1}\left(s_{0}\right), x_{n+2}\left(s_{0}\right)\right)$ and parallel to $\left[e_{1}, \ldots, e_{n}\right]$ is a sphere in case (i), a hyperboloid in case (ii) and a paraboloid in case (iii). Clearly $O\left(P^{2}\right)$ is the group of isometries of $U\left(s_{0}\right)$ and $U\left(s_{0}\right)$ is the orbit under $O\left(P^{2}\right)$ of the point $\left(x_{1}\left(s_{0}\right), 0, \ldots, 0, x_{n+1}\left(s_{0}\right), x_{n+2}\left(s_{0}\right)\right)$, i.e., $U\left(s_{0}\right)$ is the parallel of $M^{n}$ passing through this point. Thus a parametrization of $M^{n}$ can be obtained by taking a parametrization of the parallel $U\left(s_{0}\right)$ and letting $s_{0}$ vary.

For the case (i), we take $\varphi\left(t_{1}, \ldots, t_{n-1}\right)=\left(\varphi_{1}, \ldots, \varphi_{n}\right)$ as an orthogonal parametrization of the unit sphere of $\left[e_{1}, \ldots, e_{n}\right]$. It follows that

$$
\begin{gathered}
f\left(t_{1}, \ldots, t_{n-1}, s\right)=\left(x_{1}(s) \varphi_{1}, \ldots, x_{1}(s) \varphi_{n}, x_{n+1}(s), x_{n+2}(s)\right), \\
\varphi_{i}=\varphi_{i}\left(t_{1}, \ldots, t_{n-1}\right), \quad \varphi_{1}^{2}+\cdots+\varphi_{n}^{2}=1
\end{gathered}
$$

is a parametrization of a rotation hypersurface generated by a curve $x_{1}(s), x_{n+1}(s)$, $x_{n+2}(s)$ around $P^{2}=\left[e_{n+1}, e_{n+2}\right]$, when $P^{2}$ is in case (i). This will be called a spherical rotation hypersurface of $H^{n+1}(c)$. Here $g_{-1}(x, x)=x_{1}^{2}+x_{2}^{2}+\cdots+x_{n+1}^{2}$ $-x_{n+2}^{2}$, if $x=\Sigma x_{k} e_{k}$. Since the curve $x_{1}(s), x_{n+1}(s), x_{n+2}(s)$ belongs to $H^{n+1}(c)$ 
and the parameter $s$ can be chosen as its arc length, we have

$$
x_{1}^{2}(s)+x_{n+1}^{2}(s)-x_{n+2}^{2}(s)=1 / c, \quad \dot{x}_{1}^{2}(s)+\dot{x}_{n+1}^{2}(s)-\dot{x}_{n+2}^{2}(s)=1,
$$

and from that we can obtain $x_{n+1}(s)$ and $x_{n+2}(s)$ as functions of $x_{1}(s)$. In fact, we can write

$$
\begin{aligned}
& x_{n+1}(s)=\sqrt{x_{1}^{2}(s)-1 / c} \sinh \phi(s), \\
& x_{n+2}(s)=\sqrt{x_{1}^{2}(s)-1 / c} \cosh \phi(s)
\end{aligned}
$$

where $\phi(s)$ is determined by (for typographical reasons, we drop the $s$ in some of the expressions below and indicate by an upper dot the derivative relative to $s$ )

$$
1=\dot{x}_{1}^{2}+\dot{x}_{n+1}^{2}-\dot{x}_{n+2}^{2}=-\frac{x_{1}^{2} \dot{x}_{1}^{2}}{x_{1}^{2}-1 / c}+\left(x_{1}^{2}-1 / c\right) \dot{\phi}^{2}+\dot{x}_{1}^{2},
$$

or

$$
\phi(s)=\int_{0}^{s} \frac{\sqrt{-1 / c+x_{1}^{2}+\dot{x}_{1}^{2} / c}}{x_{1}^{2}-1 / c} d \sigma .
$$

Similarly, by taking $\varphi\left(t_{1}, \ldots, t_{n-1}\right)$ as an orthogonal parametrization of the "unit" hyperbolic space of $\left[e_{1}, \ldots, e_{n}\right]$, we obtain that

$$
\begin{gathered}
f\left(t_{1}, \ldots, t_{n-1}, s\right)=\left(x_{1}(s) \varphi_{1}, \ldots, x_{1}(s) \varphi_{n}, x_{n+1}(s), x_{n+2}(s)\right), \\
\varphi_{i}=\varphi_{i}\left(t_{1}, \ldots, t_{n-1}\right), \quad-\varphi_{1}^{2}+\varphi_{2}^{2}+\cdots+\varphi_{n}^{2}=-1,
\end{gathered}
$$

is a parametrization of a rotation hypersurface generated by a curve $x_{1}(s), x_{n+1}(s)$, $x_{n+2}(s)$ around $P^{2}=\left[e_{n+1}, e_{n+2}\right]$, where $P^{2}$ is in case (ii). This will be called a hyperbolic rotation hypersurface of $H^{n+1}$. Here $g_{-1}(x, x)=-x_{1}^{2}+x_{2}^{2}+\cdots+x_{n+2}^{2}$ if $x=\sum x_{k} e_{k}$, and as before we can determine $x_{n+1}(s)$ and $x_{n+2}(s)$ by

$$
x_{n+1}(s)=\sqrt{x_{1}^{2}(s)+1 / c} \sin \phi(s), \quad x_{n+2}(s)=\sqrt{x_{1}^{2}(s)+1 / c} \cos \phi(s),
$$

$$
\phi(s)=\int_{0}^{s} \frac{\sqrt{1 / c+x_{1}^{2}+\dot{x}_{1}^{2} / c}}{x_{1}^{2}+1 / c} d \sigma .
$$

Finally, to parametrize $U\left(s_{0}\right)$ in case (iii), we observe that the orbit of $\left(x_{1}\left(s_{0}\right), 0, \ldots, 0, x_{n+1}\left(s_{0}\right), x_{n+2}\left(s_{0}\right)\right)=x_{0}$ under $A_{i}$ is given by

$$
\begin{aligned}
A_{i}\left(x_{0}\right) & =(x_{1}\left(s_{0}\right), 0, \ldots, 0, \overbrace{t_{i} x_{1}\left(s_{0}\right)}^{i}, 0, \ldots,-\frac{t_{i}^{2}}{2} x_{1}\left(s_{0}\right)+x_{n+1}\left(s_{0}\right), x_{n+2}\left(s_{0}\right)) \\
= & \left(x_{1}\left(s_{0}\right), 0, \ldots, 0, t_{i} x_{1}\left(s_{0}\right), 0, \ldots,-\frac{-1 / c+x_{n+2}^{2}\left(s_{0}\right)+t_{i}^{2} x_{1}^{2}\left(s_{0}\right)}{2 x_{1}\left(s_{0}\right)}, x_{n+2}\left(s_{0}\right)\right),
\end{aligned}
$$


since $2 x_{1}\left(s_{0}\right) x_{n+1}\left(s_{0}\right)+x_{n+2}^{2}\left(s_{0}\right)=1 / c$. It follows that we can take $t_{2}, t_{3}, \ldots, t_{n}$ as parameters for $U\left(s_{0}\right)$, hence

$$
\begin{aligned}
f\left(t_{2}, t_{3}, \ldots, t_{n}, s\right)=\left(x_{1}(s),\right. & x_{1}(s) t_{2}, \ldots, x_{1}(s) t_{i}, \ldots, x_{1}(s) t_{n}, \\
& \left.-\frac{-1 / c+x_{n+2}^{2}(s)+x_{1}^{2}(s) \Sigma_{i} t_{i}^{2}}{2 x_{1}(s)}, x_{n+2}(s)\right)
\end{aligned}
$$

is a parametrization for a rotation hypersurface generated by the curve $x_{1}(s)$, $x_{n+1}(s), x_{n+2}(s)$ around $P^{2}=\left[e_{n+1}, e_{n+2}\right]$, where $P^{2}$ is the case (iii). This will be called a parabolic rotation hypersurface of $H^{n+1}$. Here $g_{-1}(x, x)=2 x_{1} x_{n+1}+x_{2}^{2}$ $+\cdots+x_{n}^{2}+x_{n+2}^{2}$ if $x=\sum x_{k} e_{k}$. To determine $x_{n+2}(s)$, we proceed as before by requiring $s$ to be the arc length of the meridian curve $x_{1}(s), x_{n+1}(s), x_{n+2}(s)$. Since

$$
2 x_{1} x_{n+1}+x_{n+2}^{2}=1 / c, 2 \dot{x}_{1} \dot{x}_{n+1}+\dot{x}_{n+2}^{2}=1 \text {, }
$$

we obtain

$$
\left(\dot{x}_{n+2} x_{1}-\dot{x}_{1} x_{n+2}\right)=\sqrt{x_{1}^{2}+\dot{x}_{1}^{2} / c}
$$

hence

$$
x_{n+2}=x_{1} \int_{0}^{s} \frac{\sqrt{x_{1}^{2}+\dot{x}_{1}^{2} / c}}{x_{1}^{2}} d \sigma .
$$

(2.9) Although we have so far restricted ourselves to the hyperbolic space, where the situation is more interesting due to the presence of case (iii), the above considerations extend trivially to other spaces of constant curvature. For the purposes of later sections, it will be convenient to have a unified notation. Thus we will denote by $R^{n}$ the space of $n$-tuples $\left(x_{1}, \ldots, x_{n}\right)=x$ with the Riemannian metric $g_{1}(x, y)=\Sigma x_{i} y_{i}, y=\left(y_{1}, \ldots, y_{n}\right)$, and write the model spaces of constant curvature as

$$
\begin{aligned}
& S^{n+1}(c)=\left\{x \in R^{n+2} ; g_{1}(x, x)=1 / c\right\}=\bar{M}^{n+1}(c), \quad c>0, \\
& H^{n+1}(c)=\left\{x \in L^{n+2} ; g_{-1}(x, x)=1 / c, x_{1}>0\right\}=\bar{M}^{n+1}(c), \quad c<0 \\
& R^{n+1}=\left\{x \in R^{n+2} ; x_{n+2}=0\right\}=\bar{M}^{n+1}(0) .
\end{aligned}
$$

We will refer to $R^{n+2}$ or $L^{n+2}$, as the case may be, as the ambient space of $\bar{M}^{n+1}(c)$. The notation $O\left(P^{2}\right)$ will again denote the set of orthogonal transformations of the ambient space that leave a given $P^{2}$ pointwise fixed. Definition (2.2) extends to this general situation if we replace $H^{n+1}(c)$ by $\bar{M}^{n+1}(c)$ and $L^{n+2}$ by the corresponding ambient space. It is then immediate that a rotation hypersurface of $\bar{M}^{n+1}(c), c>0$, can be parametrized by

$$
f\left(t_{1}, \ldots, t_{n-1}, s\right)=\left(x_{1}(s) \varphi_{1}, \ldots, x_{1}(s) \varphi_{n}, x_{n+1}(s), x_{n+2}(s)\right),
$$

where $\varphi\left(t_{1}, \ldots, t_{n-1}\right)=\left(\varphi_{1}, \ldots, \varphi_{n}\right)$ is an orthogonal parametrization of a unit sphere in $\left[e_{1}, \ldots, e_{n}\right]$. Here $P^{2}=\left[e_{n+1}, e_{n+2}\right]$. By requiring $s$ to be the arc length of 
the meridian $x_{1}(s), x_{n+1}(s), x_{n+2}(s)$ we obtain

$$
\begin{gathered}
x_{n+1}=\left(1 / c-x_{1}^{2}\right)^{1 / 2} \sin \phi, \quad x_{n+2}=\left(1 / c-x_{1}^{2}\right)^{1 / 2} \cos \phi, \\
\phi(s)=\int_{0}^{s} \frac{\left(1 / c-x_{1}^{2}-\dot{x}_{1}^{2} / c\right)^{1 / 2}}{1 / c-x_{1}^{2}} d \sigma .
\end{gathered}
$$

Similarly,

$$
f\left(t_{1}, \ldots, t_{n-1}, s\right)=\left(x_{1}(s) \varphi_{1}, \ldots, x_{1}(s) \varphi_{n}, x_{n+1}(s)\right),
$$

where $\varphi\left(t_{1}, \ldots, t_{n-1}\right)=\left(\varphi_{1}, \ldots, \varphi_{n}\right)$ is the same as before, is a parametrization of a rotation hypersurface of $R^{n+1}$; here $s$ is the arc length of the curve $x_{1}(s), x_{n+1}(s)$.

It will sometimes be expedient to use the notation $M_{c, \delta}^{n}, \delta=1,0$ or -1 , to denote a rotation hypersurface of $\bar{M}^{n+1}(c)$, where

$$
\text { if } c<0, \quad \begin{cases}\delta=1 & \text { means that } M_{c, \delta} \text { is spherical, } \\ \delta=0 & \text { means that } M_{c, \delta} \text { is parabolic, } \\ \delta=-1 & \text { means that } M_{c, \delta} \text { is hyperbolic, }\end{cases}
$$

and if $c \geqslant 0$, then $\delta=1$.

Unless otherwise stated, all manifolds are connected and $C^{\infty}$.

3. The principal curvatures; rotation hypersurfaces with constant mean curvature.

(3.1) In this section we compute the principal curvatures of a rotation hypersurface. This will allow us to establish a second order ordinary differential equation for rotation hypersurfaces with constant mean curvature. In general, for each type $M_{c, \delta}^{n}$ there exists a one-parameter family of distinct solutions of the equation. We will prove, however, that for the parabolic case, the solution is unique up to isometries. We will also prove, for $n=2$, that the complete minimal rotation surfaces of $\bar{M}^{3}(c)$ are embedded if $c<0$, and that their universal coverings are conjugate to the geodesically ruled minimal surfaces in $\bar{M}^{3}(c)$.

(3.2) Proposition. Let $M_{c, \delta}^{n} \subset \bar{M}^{n+1}(c)$ be a rotation hypersurface. Then the directions of the parameters described in $\$ 1$ are principal directions, the principal curvatures along the coordinate curves $t_{i}$ are all equal and given by

$$
\lambda=-\frac{\sqrt{\delta-c x_{1}^{2}-\dot{x}_{1}^{2}}}{x_{1}}
$$

and the principal curvature along the coordinate curve $s$ is

$$
\mu=\frac{\ddot{x}_{1}+c x_{1}}{\sqrt{\delta-c x_{1}^{2}-\dot{x}_{1}^{2}}} .
$$

Thus $\lambda$ has multiplicity at least equal to $n-1$.

Proof. We give the proof for the cases: (1) $c<0, \delta=-1$; (2) $c<0, \delta=0$. All the other cases will be similar to Case 1 . 
Case (1). Since, from (2.7),

$$
\begin{aligned}
& \frac{\partial f}{\partial s}=\left(\dot{x}_{1} \varphi_{1}, \ldots, \dot{x}_{1} \varphi_{n}, \dot{x}_{n+1}, \dot{x}_{n+2}\right), \\
& \frac{\partial f}{\partial t_{j}}=\left(x_{1} \frac{\partial \varphi_{1}}{\partial t_{j}}, \ldots, x_{1} \frac{\partial \varphi_{n}}{\partial t_{j}}, 0,0\right)
\end{aligned}
$$

and $\varphi$ is an orthogonal parametrization, we obtain

$$
g_{-1}\left(\frac{\partial f}{\partial s}, \frac{\partial f}{\partial s}\right)=1, \quad g_{-1}\left(\frac{\partial f}{\partial s}, \frac{\partial f}{\partial t_{i}}\right)=0, \quad g_{-1}\left(\frac{\partial f}{\partial t_{i}}, \frac{\partial f}{\partial t_{j}}\right)=\alpha_{i j} x_{1}^{2}
$$

where we have set $\alpha_{i j}=\Sigma_{k}\left\langle\partial \varphi_{k} / \partial t_{i}, \partial \varphi_{k} / \partial t_{j}\right\rangle$. A unit normal field $N$ is given by

$$
N=\sqrt{-c}\left(\varphi\left(\dot{x}_{n+2} x_{n+1}-\dot{x}_{n+1} x_{n+2}\right),\left(\dot{x}_{n+2} x_{1}-\dot{x}_{1} x_{n+2}\right),\left(\dot{x}_{1} x_{n+1}-\dot{x}_{n+1} x_{1}\right)\right)
$$

and

$$
\frac{\partial^{2} f}{\partial s^{2}}=\left(\ddot{x}_{1} \varphi, \ddot{x}_{n+1}, \ddot{x}_{n+2}\right), \quad \frac{\partial^{2} f}{\partial s \partial t_{j}}=\left(\dot{x}_{1} \frac{\partial \varphi}{\partial t_{j}}, 0,0\right), \quad \frac{\partial^{2} f}{\partial t_{i} \partial t_{j}}=\left(x_{1} \frac{\partial^{2} \varphi}{\partial t_{i} \partial t_{j}}, 0,0\right) .
$$

It follows from the above that the coordinate curves are lines of curvature. The principal curvatures along the coordinate curves $t_{i}$ are given by

$$
\lambda=-\frac{\sqrt{-c}}{x_{1}}\left(\dot{x}_{n+2} x_{n+1}-\dot{x}_{n+1} x_{n+2}\right)=-\frac{\sqrt{-1-c x_{1}^{2}-\dot{x}_{1}^{2}}}{x_{1}},
$$

where we used the fact that

$$
x_{n+2}=\left(x_{1}^{2}+1 / c\right)^{1 / 2} \cos \phi \text { and } x_{n+1}=\left(x_{1}^{2}+1 / c\right)^{1 / 2} \sin \phi .
$$

This proves the first part of Proposition (3.2).

It is now an easy matter to compute $\mu$ and find

$$
\begin{aligned}
\mu=\sqrt{-c}\left\{\ddot{x}_{1}\left(\dot{x}_{n+2} x_{n+1}-\dot{x}_{n+1} x_{n+2}\right)+\ddot{x}_{n+1}\left(\dot{x}_{n+2} x_{1}-\dot{x}_{1} x_{n+2}\right)\right. & \left.\quad+\ddot{x}_{n+2}\left(\dot{x}_{1} x_{n+1}-x_{1} \dot{x}_{n+1}\right)\right\}, \\
=\sqrt{-c}\left\{\ddot{x}_{1}\left(\dot{x}_{n+2} x_{n+1}-\dot{x}_{n+1} x_{n+2}\right)+\dot{x}_{1}\left(\ddot{x}_{n+2} x_{n+1}-x_{n+2} \ddot{x}_{n+1}\right)\right. & \left.+x_{1}\left(\dot{x}_{n+2} \ddot{x}_{n+1}-\ddot{x}_{n+2} \dot{x}_{n+1}\right)\right\}
\end{aligned}
$$

and this completes the proof for Case (1).

Case (2). From (2.8), we see that

$$
\begin{aligned}
& \frac{\partial f}{\partial t_{i}}=(0,0, \ldots, 0, \overbrace{x_{1}}^{i}, 0, \ldots, 0,-x_{1} t_{i}, 0), \\
& \frac{\partial f}{\partial s}=\left(\dot{x}_{1}, \dot{x}_{1} t_{2}, \ldots, \dot{x}_{1} t_{n},-\frac{2 x_{1} x_{n+2} \dot{x}_{n+2}+x_{1}^{2} \dot{x}_{1} \Sigma t_{i}^{2}+\dot{x}_{1} / c-\dot{x}_{1} x_{n+2}}{2 x_{1}^{2}}, \dot{x}_{n+2}\right) .
\end{aligned}
$$


It follows that

$$
\left\{\begin{array}{l}
g_{-1}\left(\frac{\partial f}{\partial t_{i}}, \frac{\partial f}{\partial t_{j}}\right)=x_{1}^{2} \delta_{i j}, \quad g_{-1}\left(\frac{\partial f}{\partial t_{i}}, \frac{\partial f}{\partial s}\right)=0 \\
g_{-1}\left(\frac{\partial f}{\partial s}, \frac{\partial f}{\partial s}\right)=\frac{\left(x_{n+2} \dot{x}_{1}-x_{1} \dot{x}_{n+2}\right)^{2}-\dot{x}_{1}^{2} / c}{x_{1}^{2}}=\frac{r-\dot{x}_{1}^{2} / c}{x_{1}^{2}}=1
\end{array}\right.
$$

where we have set $r=\left(x_{n+2} \dot{x}_{1}-x_{1} \dot{x}_{n+2}\right)^{2}$ and used the fact that the meridians are parametrized by arc length.

To find a normal vector to $M^{n}$, we write

$$
\eta=\zeta+\frac{\dot{x}_{1}}{x_{1} c} \frac{\partial f}{\partial s}, \quad \zeta=\left(x_{1}, x_{1} t_{2}, \ldots, x_{1} t_{n}, \frac{-1 / c-x_{n+2}^{2}-x_{1}^{2} \Sigma t_{i}^{2}}{2 x_{1}}, x_{n+2}\right) .
$$

It is easily checked that $g_{-1}(\zeta, \zeta)=-1 / c$ and that

$$
g_{-1}(\zeta, f)=0, \quad g_{-1}\left(\zeta, \frac{\partial f}{\partial t_{i}}\right)=0, \quad g_{-1}\left(\zeta, \frac{\partial f}{\partial s}\right)=-\frac{\dot{x}_{1}}{x_{1} c}
$$

Thus $\eta$ is a normal vector and $g_{-1}(\eta, \eta)=-r / c x_{1}^{2}$. By noticing that $\partial^{2} f / \partial t_{i}^{2}=$ $\left(0,0, \ldots, 0,-x_{1}, 0\right)$, we obtain

$$
\left\|\frac{\partial f}{\partial t_{i}}\right\|^{-2} g_{-1}\left(\frac{\partial^{2} f}{\partial t_{i}^{2}}, \eta\right)=\frac{1}{x_{1}^{2}}\left\{-x_{1}^{2}-\frac{\dot{x}_{1}^{2}}{c}\right\}=-\frac{r}{x_{1}^{2}} .
$$

On the other hand,

$$
g_{-1}\left(\frac{\partial^{2} f}{\partial s^{2}}, \eta\right)=-g_{-1}\left(\frac{\partial f}{\partial s}, \frac{\partial \eta}{\partial s}\right)=-g_{-1}\left(\frac{\partial f}{\partial s}, \frac{\partial \zeta}{\partial s}\right)-\left(\frac{\dot{x}_{1}}{c x_{1}}\right)-\frac{\dot{x}_{1}}{c x_{1}} g_{-1}\left(\frac{\partial f}{\partial s}, \frac{\partial^{2} f}{\partial s^{2}}\right)
$$

To simplify the above expression, we observe that, since $g_{-1}(\partial f / \partial s, \partial f / \partial s)=1$, we have

$$
0=\frac{d}{d s} g_{-1}\left(\frac{\partial f}{\partial s}, \frac{\partial f}{\partial s}\right)=2 g_{-1}\left(\frac{\partial f}{\partial s}, \frac{\partial^{2} f}{\partial s^{2}}\right)
$$

Furthermore,

$$
\frac{\partial \zeta}{\partial s}=\left(\dot{x}_{1}, \dot{x}_{1} t_{2}, \ldots, \dot{x}_{1} t_{n}, \frac{-2 x_{1} x_{n+2} \dot{x}_{n+2}-x_{1}^{2} \dot{x}_{1} \Sigma t_{i}^{2}+\dot{x}_{1} / c+\dot{x}_{1} x_{n+2}^{2}}{2 x_{1}^{2}}, \dot{x}_{n+2}\right),
$$

hence $g_{-1}(\partial \zeta / \partial s, \partial f / \partial s)=r / x_{1}^{2}$. Thus (3.7) becomes

$$
g_{-1}\left(\frac{\partial^{2} f}{\partial s^{2}}, \eta\right)=-\frac{r}{x_{1}^{2}}-\frac{\ddot{x}_{1} x_{1}-\dot{x}_{1}^{2}}{x_{1}^{2} c}
$$


It follows that

$$
\begin{gathered}
\lambda=-\frac{\sqrt{-c r}}{x_{1}}=-\frac{\sqrt{-c x_{1}^{2}-\dot{x}_{1}^{2}}}{x_{1}}, \\
\mu=\left(-\frac{r}{x_{1}^{2}}-\frac{\ddot{x}_{1} x_{1}-\dot{x}_{1}^{2}}{x_{1}^{2} c}\right) \frac{x_{1} \sqrt{-c}}{\sqrt{r}}=\frac{\ddot{x}_{1}+c x_{1}}{\sqrt{-c x_{1}^{2}-\dot{x}_{1}^{2}}},
\end{gathered}
$$

and this proves Proposition (3.2) for Case (2).

(3.8) COROLlaRY. The first and second fundamental forms of $M_{c, \delta}$ are given by (cf. (3.5), (3.6))

(3.9) $\mathrm{I}=d s^{2}+x_{1}^{2}(s) \sum_{i} \alpha_{i i} d t_{i}^{2}$,

(3.10) $\mathrm{II}=\mu(s) d s^{2}+\lambda(s) x_{1}^{2}(s) \sum_{i} \alpha_{i i} d t_{i}^{2}$,

respectively, where $\alpha_{i i}=1$ if $\delta=0$.

(3.11) REMARK. One might question whether $\mu$ is well defined at points where $\lambda=0$. To see that this is the case, let us consider the spherical rotation hypersurfaces $(\delta=1)$ of $H^{n+1}(c)$. From $(2.6)^{\prime}$, it follows that

$$
e^{\phi(s)}=\frac{x_{n+1}}{\sqrt{x_{1}^{2}(s)-1 / c}}+\frac{x_{n+2}}{\sqrt{x_{1}^{2}(s)-1 / c}},
$$

whence $\phi(s)$ is a differentiable function. From (2.6)" we obtain that

$$
\ddot{\phi}(s)=\frac{\dot{x}_{1}\left(x_{1}+\ddot{x} / c\right)}{\left(x_{1}^{2}-1 / c\right) \sqrt{-1 / c+x_{1}^{2}+\dot{x}_{1}^{2} / c}}+\frac{\sqrt{-1 / c+x_{1}^{2}+\dot{x}_{1}^{2} / c}}{\left(x_{1}^{2}-1 / c\right)^{2}} 2 x_{1} \dot{x}_{1} .
$$

Now $\lambda\left(s_{0}\right)=0$ implies that $\dot{x}_{1}\left(s_{0}\right) \neq 0$. Thus

$$
\ddot{\phi}\left(s_{0}\right)=\frac{\dot{x}_{1}\left(s_{0}\right)}{\left(x_{1}^{2}\left(s_{0}\right)-1 / c\right)} \frac{\ddot{x}_{1}\left(s_{0}\right)+c x_{1}\left(s_{0}\right)}{\sqrt{1-c x_{1}^{2}\left(s_{0}\right)-\dot{x}_{1}^{2}\left(s_{0}\right)}}=\frac{\dot{x}_{1}\left(s_{0}\right)}{x_{1}^{2}\left(s_{0}\right)-1 / c} \mu\left(s_{0}\right) .
$$

Therefore, $\mu\left(s_{0}\right)$ is well defined and given by (3.4). The remaining cases are similar.

(3.12) REMARK. For the case $n=2$, it is easily seen that we can choose $t_{1}=t$ so that $\alpha_{11}=1$. Next, by replacing the parameter $s$ by $\sigma=\int_{0}^{s} d s / x_{1}(s)$, we obtain that the first quadratic form $\mathrm{I}$ in the parameters $(\sigma, t)$ is given by $\mathrm{I}=x_{1}^{2}\left(d \sigma^{2}+d t^{2}\right)$, hence the parameters $(\sigma, t)$ are isothermal.

From Proposition (3.2), we can write down a differential equation to be satisfied by a rotation hypersurface $M_{c, \delta}$ with a prescribed function $H=H(s)$ as the mean curvature, namely

$$
x_{1} \ddot{x}_{1}+(n-1) \dot{x}_{1}^{2}+n c x_{1}^{2}-\delta(n-1)=n H x_{1} \sqrt{\delta-c x_{1}^{2}-\dot{x}_{1}^{2}} .
$$

For $H \equiv 0, c=0$, and $n=2$, (3.13) agrees with the equation of a catenary parametrized by arc length. Therefore we will call catenoids the solutions of (3.13) for $H \equiv 0$, and will distinquish, for $c<0$, the spherical, parabolic and hyperbolic catenoids. For $c>0$, rotation minimal hypersurfaces were determined in a slightly 
different way by Otsuki [10]. It should be noticed that the Clifford minimal hypersurface $S^{1} \times S^{n-1} \subset S^{n+1}(1)$ corresponds to the trivial solution $x_{1}^{2}=$ $(n-1) / n$ of (3.13), when $H=0, \delta=1, c=1$.

We want to use (3.13) to prove the following fact.

(3.14) THEOREM. Up to isometries, there exists a unique, complete, parabolic rotation hypersurface of $H^{n+1}(c)$ with a given constant mean curvature $H$. Such a hypersurface is simply-connected and if $H \equiv 0$ it is embedded.

For the proof we need the following lemma.

(3.15) LeMma. Let $H$ be constant. Then a first integral of (3.13) is given by

$$
\dot{x}_{1}^{2}=\delta-c x_{1}^{2}-\left(H x_{1}-\frac{a}{x_{1}^{n-1}}\right)^{2}, \quad a=\text { const. }
$$

Proof of Lemma (3.15). Set $y=\sqrt{\delta-c x_{1}^{2}-\dot{x}_{1}^{2}}$. Then $y \dot{y}=-\dot{x}_{1}\left(c x_{1}+\ddot{x}_{1}\right)$, and by using the value of $\ddot{x}_{1}$ from (3.13) we obtain

$$
\dot{y}=-\frac{\dot{x}_{1}}{x_{1}}\left[(n-1) y+n x_{1} H\right] \text {. }
$$

Now set $f=y+H x_{1}$. Since $H$ is constant, the above implies that $\dot{f}_{1}=-(n-1) f \dot{x}_{1}$ which yields that $f=a / x_{1}^{n-1}$, where $a$ is a constant. It follows that

$$
y^{2}=\left(f-H x_{1}\right)^{2}=\delta-c x_{1}^{2}-\dot{x}_{1}^{2}
$$

which gives (3.16). Q.E.D.

Proof of Theorem (3.14). Set $\delta=0$ and $x_{1}^{n}=z$ in (3.15). Then

$$
\dot{z}^{2}=n^{2}\left[-\left(c+H^{2}\right) z^{2}+2 a H z-a^{2}\right]
$$

which yields, by adjusting the origin of the parameter $s$,

$$
s=\frac{1}{n} \int \frac{d z}{\sqrt{-\left(c+H^{2}\right) z^{2}+2 H a z-a^{2}}}=g\left(x_{1}\right) .
$$

Let $x_{1}=x_{1}(s)$ be the inverse function of $g$, and define $\tilde{x}_{1}(s)$ by $x_{1}(s)=a^{1 / n} \tilde{x}_{1}(s)$. We claim that $\tilde{x}_{1}(s)$ does not depend on $a$. This follows from the fact that, by setting $\tilde{x}_{1}^{n}=\tilde{z}$, we obtain

$$
s=\frac{1}{n} \int \frac{d z}{\sqrt{-\left(c+H^{2}\right) z^{2}+2 H a z-a^{2}}}=\frac{1}{n} \int \frac{d \tilde{z}}{\sqrt{-\left(c+H^{2}\right) \tilde{z}^{2}+2 H \tilde{z}-1}}
$$

and this proves our claim.

Now, by inserting $x_{1}(s)=a^{1 / n} \tilde{x}_{1}(s)$ in the values of $\lambda$ and $\mu$ given by (3.3) and (3.4) (with $\delta=0$ ), we see that $\lambda$ and $\mu$ do not depend on $a$ either. By looking at the first and second fundamental forms:

$$
\begin{gathered}
\mathrm{I}=d s^{2}+\tilde{x}_{1}^{2}(s) a^{2 / n} \sum_{i} d t_{i}^{2}, \\
\mathrm{II}=\mu(s) d s^{2}+\lambda(s) \tilde{x}_{1}^{2} a^{2 / n} \sum_{i} d t_{i}^{2},
\end{gathered}
$$


we notice that by changing the parameter $t_{i}$ into $a^{1 / n} t_{i}=\bar{t}_{i}$, the expressions of I and II in the parameters $\left(s, \bar{t}_{i}\right)$ do not depend on $a$. Since a simply-connected hypersurface in $H^{n+1}(c)$ is determined up to isometries by I and II, this proves the first part of the theorem.

To prove the second part, we can assume that $c=-1$. Then set $H=0, c=-1$ and $a=1$ in (3.17) to obtain by integration $x_{1}(s)=(\cosh n s)^{1 / n}$. By observing that $x_{1}(s)$ is unbounded, that for $s \neq 0, \dot{x}_{1}(s) \neq 0$, and that $x_{1}\left(s_{1}\right)=x_{1}\left(s_{2}\right)$ implies $s_{2}= \pm s_{1}$, we are reduced to prove that $x_{n+2}(s) \neq x_{n+2}(-s)$, for all $s \neq 0$.

To see that, assume that the contrary holds and use (2.8)' to obtain

$$
\left(\dot{x}_{n+2} x_{1}-\dot{x}_{1} x_{n+2}\right)^{2}=x_{1}^{2}-\dot{x}_{1}^{2}=(\cosh n s)^{2 / n-2} \text {, }
$$

hence

$$
x_{n+2} \sinh n s-\dot{x}_{n+2} \cosh n s= \pm 1 .
$$

We can assume that the right-hand side of (3.18) is - 1 . It is easily checked that the solution $x_{n+2}(s)$ of $(3.18)$ that satisfies $x_{n+2}(0)=0$ has the property that $x_{n+2}(s)$ is an odd function of $s$. Thus, there exists by hypothesis an $s=s_{0}$ such that

$$
x_{n+2}\left( \pm s_{0}\right)=0, \quad \dot{x}_{n+2}\left( \pm s_{0}\right)>0, \quad x_{n+2}(0)=0, \quad \dot{x}_{n+2}(0)=1,
$$

and $x_{n+2}(s)$ is not zero in the open intervals $\left(-s_{0}, 0\right),\left(0, s_{0}\right)$. This is clearly a contradiction and completes the proof of Theorem (3.14).

For the case $n=2$ and $H=0,(3.13)$ can be easily integrated in terms of elementary functions. Since we want to use some of the expressions so obtained to prove Theorems (3.26) and (3.31) below, we will give some details. For the rest of this section, we will assume, for simplicity, that $c=1$ or $c=-1$.

For $c=-1,(3.13)$ becomes

$$
x_{1} \ddot{x}_{1}+\dot{x}_{1}^{2}=2 x_{1}^{2}+\delta .
$$

By setting $2 x_{1}^{2}+\delta=y$, we see that $\ddot{y}=4 y$; hence, by adjusting the origin of the parameter $s$, we can write the solutions of (3.19) as

$$
x_{1}(s)=(a \cosh 2 s-\delta / 2)^{1 / 2}, \quad a=\text { const. }
$$

The remaining coordinates of the meridian curve are then given by (3.18), if $\delta=0$, and (see (2.6)' and (2.7)')

$$
x_{3}(s)=\left(x_{1}^{2}(s)+1\right)^{1 / 2} \sinh \phi(s), \quad x_{4}(s)=\left(x_{1}^{2}(s)+1\right)^{1 / 2} \cosh \phi(s) \quad \text { if } \delta=1,
$$

$$
x_{3}(s)=\left(x_{1}^{2}(s)-1\right)^{1 / 2} \sin \phi(s), \quad x_{4}(s)=\left(x_{1}^{2}(s)-1\right)^{1 / 2} \cos \phi(s) \quad \text { if } \delta=-1,
$$

where $\phi$ is given by (see (2.6)" and $\left.(2.7)^{\prime \prime}\right)$

$$
\begin{aligned}
\phi(s) & =\int_{0}^{s} \frac{\left(\delta+x_{1}^{2}-\dot{x}_{1}^{2}\right)^{1 / 2}}{x_{1}^{2}+\delta} d s \\
& =\left(a^{2}-1 / 4\right)^{1 / 2} \int_{0}^{s}(a \cosh 2 s+\delta / 2)^{-1}(a \cosh 2 s-\delta / 2)^{-1 / 2} d s
\end{aligned}
$$


It follows from (3.23) that $a>1 / 2$ if $\delta= \pm 1$, and as we have seen in the proof of Theorem (3.14), $a=1$ if $\delta=0$.

For $c=1$, it is easily seen that the solutions of (3.13) are

$$
x_{1}(s)=(1 / 2+a \cos 2 s)^{1 / 2}, \quad a=\text { const. }
$$

In this case, the remaining coordinates of the meridian curve are given by $(2.10)^{\prime}$, where $\phi$ is ( $\left.\operatorname{see}(2.10)^{\prime \prime}\right)$

$$
\begin{aligned}
\phi(s) & =\int_{0}^{s} \frac{\left(1-x_{1}^{2}-\dot{x}_{1}^{2}\right)^{1 / 2}}{1-x_{1}^{2}} d s \\
& =\left(1 / 4-a^{2}\right)^{1 / 2} \int_{0}^{s}(1 / 2+a \cos 2 s)^{-1 / 2}(1 / 2-a \cos 2 s)^{-1} d s .
\end{aligned}
$$

It follows from (3.24) that for $c=1,-1 / 2<a<1 / 2$.

We are now in position to prove the following result.

(3.26) THEOREM. Let $f: M^{2} \rightarrow H^{3}(-1)$ be a complete catenoid. Then $f$ is an embedding.

Proof. The case $\delta=0$ is contained in Theorem (3.14); thus we can assume that $\delta= \pm 1$. By arguing as in the last part of the proof of Theorem (3.14), we are reduced to proving that either $x_{3}$ or $x_{4}$ satisfies the condition

$$
x_{2+i}(s) \neq x_{2+i}(-s) \text { for all } s \neq 0, i=1 \text { or } i=2 \text {. }
$$

To see that this is the case, we first observe from (3.23) that $\phi(s)$ is an odd function of $s$. Since the hyperbolic sine is injective, we see that $x_{3}(s)$ of (3.21) satisfies (3.27). This proves Theorem (3.26) for $\delta=1$. For $\delta=-1$, we observe that $\phi^{\prime}(s)>0$ for all $s$, and, by using a computer, we check that $\phi(\infty)<\pi$ for all $a>1 / 2$. It follows that $x_{3}(s)$ of (3.22) satisfies (3.27) and this concludes the proof.

We now want to use the above explicit expressions to prove that the conjugate surfaces to the two-dimensional catenoids in $\bar{M}^{3}(c)$ are the geodesically-ruled minimal surfaces in $\bar{M}^{3}(c)$.

We recall that the geodesically-ruled minimal surfaces (see Lawson [7, p. 352]) of $M^{3}(c)$ can be parametrized by

$$
f(s, t)=(\cos \lambda t \cos s, \sin \lambda t \sin s, \cos t \sin s, \sin t \sin s)
$$

if $c=1$, and by

(3.29) $f(s, \bar{t})=(\cosh \lambda \bar{t} \cosh s, \sinh \lambda \bar{t} \cosh s, \cos \bar{t} \sinh s, \sin \bar{t} \sinh s)$

if $c=-1$; here $\lambda$ is a positive real number (the parametrization (3.29) is not explicitly given in Lawson [7] but the same proof of (3.28) applies). It will be convenient to set in (3.29) $\lambda=\alpha / \beta$ and to change the parameter $t$ to $\bar{t}=\beta t$. Thus (3.29) can be written as

(3.29)' $f(s, t)=(\cosh \alpha t \cosh s, \sinh \alpha t \cosh s, \cos \beta t \sinh s, \sin \beta t \sinh s)$.

We also recall (Lawson [7], see the proof of Theorem 8, p. 366) the following fact: Let $f: M^{2} \rightarrow \bar{M}^{3}(c)$ be a minimal surface in isothermal parameters $(\sigma, t)$. Denote by $\mathrm{I}=E\left(d \sigma^{2}+d t^{2}\right)$ and by $\mathrm{II}=\beta_{11} d \sigma^{2}+2 \beta_{12} d \sigma d t+\beta_{22} d t^{2}$ the first and second 
fundamental forms of $f$, respectively. Set $\psi=\beta_{11}-i \beta_{12}$ and define a family of quadratic forms depending on a parameter $\theta, 0 \leqslant \theta \leqslant 2 \pi$, by

$$
\beta_{11}(\theta)=\operatorname{Re}\left\{e^{i \theta} \psi\right\}, \quad \beta_{22}(\theta)=-\operatorname{Re}\left\{e^{i \theta} \psi\right\}, \quad \beta_{12}(\theta)=\operatorname{Im}\left\{e^{i \theta} \psi\right\} .
$$

Then $\mathrm{I}_{\theta}=\mathrm{I}$ and $\mathrm{II}_{\theta}=\beta_{11}(\theta) d \sigma^{2}+2 \beta_{12}(\theta) d \sigma d t+\beta_{22}(\theta) d t^{2}$ satisfy the Gauss and Codazzi equations, thus giving rise to an isometric family $f_{\theta}: \tilde{M}^{2} \rightarrow \bar{M}^{3}(c)$ of minimal immersions; here $\tilde{M}^{2}$ is the universal covering of $M^{2}$. The immersion $f_{\pi / 2}$ is called the conjugate immersion to $f_{0}=f$.

(3.31) THEOREM. Let $f: M^{2} \rightarrow H^{3}(-1)$ be a catenoid given by (3.21). Its conjugate minimal surface is the geodesically-ruled minimal surface given by (3.29)' where

$$
\left\{\begin{array}{lll}
\text { if } \delta=1, & \alpha=(a-1 / 2)^{1 / 2}, & \beta=(a+1 / 2)^{1 / 2}, \\
\text { if } \delta=0, & \alpha=\beta=1, & \\
\text { if } \delta=-1, & \alpha=(a+1 / 2)^{1 / 2}, & \beta=(a-1 / 2)^{1 / 2} .
\end{array}\right.
$$

Proof. It is easily computed that the first and second fundamental forms of the catenoid (3.20) are given by

$$
\begin{aligned}
\mathrm{I} & =d s^{2}+(a \cosh 2 s-\delta / 2) d t^{2}, \\
\mathrm{II} & =\frac{\left(a^{2}-|\delta| / 4\right)}{a \cosh 2 s-\delta / 2} d s^{2}-\left(a^{2}-|\delta| / 4\right)^{1 / 2} d t^{2},
\end{aligned}
$$

respectively. Set

$$
\sigma=\int_{0}^{s} \frac{d s}{(a \cosh 2 s-\delta / 2)^{1 / 2}} .
$$

Then

$$
\mathrm{I}=(a \cosh 2 s-\delta / 2)\left(d \sigma^{2}+d t^{2}\right), \quad \mathrm{II}=\left(a^{2}-|\delta| / 4\right)^{1 / 2}\left(d \sigma^{2}-d t^{2}\right),
$$

where $s=s(\sigma)$ is the function of $\sigma$ obtained from (3.33). Furthermore, the corresponding fundamental forms of the geodesically-ruled surface (3.29)' are

$$
\begin{aligned}
& \tilde{\mathrm{I}}=d s^{2}+\left(\beta^{2} \sinh ^{2} s+\alpha^{2} \cosh ^{2} s\right) d t^{2}, \\
& \widetilde{\mathrm{II}}=\frac{\alpha \beta}{\beta^{2} \sinh ^{2} s+\alpha^{2} \cosh ^{2} s} d s d t .
\end{aligned}
$$

Now, by using the correspondence (3.32), one sees that

$$
\begin{aligned}
a \cosh 2 s-\delta / 2 & =(a+\delta / 2)\left\{\frac{a-\delta / 2}{a+\delta / 2} \cosh ^{2} s+\sinh ^{2} s\right\} \\
& =\alpha^{2} \cosh ^{2} s+\beta^{2} \sinh ^{2} s .
\end{aligned}
$$

It follows that by making the change of parameter (3.33), we obtain

$$
\tilde{\mathrm{I}}=(a \cosh 2 s-\delta / 2)\left(d \sigma^{2}+d t^{2}\right), \quad \tilde{\mathrm{II}}=(a-|\delta| / 4)^{1 / 2} d \sigma d t .
$$

By applying the above quoted Lawson's result to the present case $\left(\beta_{12}=0\right.$, $\beta_{11}=-\beta_{22}$ ) and the fact that surfaces are uniquely determined by their first and second fundamental forms, we complete the proof. 
(3.34) REMARK. A similar correspondence can easily be established between the catenoids of $S^{3}(1)$ given by (3.24) and the geodesically-ruled surfaces (3.28) (as expected, the minimal Clifford torus appears as a self-conjugate surface). That they are conjugate has already been established by Lawson [7] by using certain reflection properties of the conjugate surface. Lawson's method can probably be applied to the hyperbolic space. The point of Theorem (3.31), however, is to give the explicit correspondence (3.32) and to show that the hyperbolic and parabolic catenoids appear naturally in this context.

(3.35) REMARK. It is possible to determine explicitly the associated family $f_{\theta}$ of the catenoids in $\bar{M}^{3}(c)$ but we will not go into that here. Also Lawson's correspondence is much more general than the one quoted above and can be stated as follows: Given an immersion $f: M^{2} \rightarrow \bar{M}^{3}(c)$ with constant mean curvature $H$, there exists a 2-parameter family of immersions $f_{\theta, \bar{c}}: \tilde{M}_{1} \rightarrow M^{3}(\bar{c}), \bar{c} \leqslant H^{2}+c, 0 \leqslant \theta \leqslant 2 \pi$, that are isometric to $f=f_{0, c}$ and have constant mean curvature $\bar{H}=\left(H^{2}+c-\bar{c}\right)^{1 / 2}$ (this is not actually stated in Theorem 8 of Lawson [7] but is contained in its proof). In the applications of the above theorem, one has to be a bit careful because the construction of the family $f_{\theta, \bar{c}}$ is given in terms of isothermal parameters, which are not always present in a natural parametrization. In any case, using these ideas we have been able to determine all surfaces of constant mean curvature in $R^{3}$ that are invariant by helicoidal motions of $R^{3}$; these include the classical rotation surfaces of constant mean curvature of Delaunay [5]. We will discuss that somewhere else.

(3.36) REMARK. Before concluding this section, it may be convenient to observe that catenoids that are not totally geodesic have no umbilic points. This follows from Lemma (3.15). In fact, by setting $H=0$ in (3.16), we obtain

$$
\delta-c x_{1}^{2}-\dot{x}_{1}^{2}=\frac{a^{2}}{\left(x_{1}^{n-1}\right)^{2}}, \quad a=\text { const. }
$$

hence, by (3.3), $\lambda^{2} x_{1}^{2}=a^{2} /\left(x_{1}^{n-1}\right)^{2}$. Since $x_{1}>0$, we can assume that $a \neq 0$; otherwise $\lambda \equiv 0$ and, since $H \equiv 0, \mu \equiv 0$ and the catenoid is totally geodesic. Thus $\lambda \neq 0$ everywhere, and there are no umbilic points.

\section{Sufficient conditions.}

(4.1) In this section we want to describe some sufficient conditions for a hypersurface of $\bar{M}^{n+1}(c), n \geqslant 3$, to be a rotation hypersurface. The main theorem is as follows.

(4.2) THEOREM. Let $f: M^{n} \rightarrow \bar{M}^{n+1}(c), n \geqslant 3$, be an arbitrary hypersurface. Assume that the principal curvatures $k_{1}, \ldots, k_{n}$ of $f$ satisfy $k_{1}=k_{2}=\cdots=k_{n-1}=-\lambda \neq 0$, $k_{n}=-\mu=-\mu(\lambda)$, and $\lambda-\mu \neq 0$. Then $f\left(M^{n}\right)$ is contained in a rotation hypersurface.

Proof. It is known (Ryan [11, p. 372]) that $M$ is foliated by the leaves of the (involutive) distributions $D_{\lambda}$ and $D_{\mu}$ determined by the eigenvectors corresponding to $\lambda$ and $\mu$, respectively; furthermore, $\lambda$ is constant along the leaves of $D_{\lambda}$. Let $p \in M$ and denote by $\Sigma_{p}$ and $L_{p}$ the leaves of $D_{\lambda}$ and $D \mu$, respectively, passing through $p$. Choose, in a neighborhood $U$ of $p$, coordinates $u_{1}, \ldots, u_{n-1}$ and $t$ such that 
$u_{1}, \ldots, u_{n-1}$ are local coordinates in $\Sigma_{p}$ and $t$ is a coordinate in $L_{p}$. Set $V_{i}=\partial / \partial u_{i}$, $T=\partial / \partial t$, and denote by $N$ a unit normal vector of the immersion $f$. We will show that, in a neighborhood of $p, f$ is a rotation hypersurface.

We will first show that $\Sigma_{p}$ is umbilic in $\bar{M}^{n+1}(c)$. Let $\bar{\nabla}$ denote the covariant derivative of $\bar{M}^{n+1}(c)$. Then

$$
\bar{\nabla}_{V_{i}} N=\lambda V_{i}, \quad \bar{\nabla}_{T} N=\mu T .
$$

Since $V_{i}(\lambda)=0$ and $\mu=\mu(\lambda), V_{i}(\mu)=0$, and

$$
\bar{\nabla}_{T} \bar{\nabla}_{V_{i}} N=\lambda^{\prime} V_{i}+\lambda \bar{\nabla}_{T} V_{i}, \quad \bar{\nabla}_{V_{i}} \bar{\nabla}_{T} N=\mu \bar{\nabla}_{V_{i}} T .
$$

From the fact that $\bar{M}^{n+1}(c)$ has constant sectional curvature and $\left[V_{i}, T\right]=0$, we conclude that

$$
0=\left(\bar{\nabla}_{T} \bar{\nabla}_{V_{i}}-\bar{\nabla}_{V_{i}} \bar{\nabla}_{T}\right) N=\lambda^{\prime} V_{i}+(\lambda-\mu) \bar{\nabla}_{V_{i}} T .
$$

Thus, since $p$ is not umbilic,

$$
\bar{\nabla}_{V_{i}} N=\lambda V_{i}, \quad \bar{\nabla}_{V_{i}} T=-\frac{\lambda^{\prime}}{\lambda-\mu} V_{i},
$$

hence $\Sigma_{p}$ is umbilic in $\bar{M}^{n+1}(c)$.

It follows that $\Sigma_{p} \subset \bar{P}^{n} \cap \bar{M}^{n+1}(c)$, where $\bar{P}^{n}$ is an affine $n$-subspace of the ambient space. We want to show that the spaces $\bar{P}^{n}$ corresponding to distinct leaves $\Sigma$ are parallel in the ambient space.

Let $x$ be the position vector of $\bar{M}^{n+1}(c)$ in the ambient space and let $\nabla$ and $g_{\varepsilon}$, $\varepsilon=1$ or -1 , be the covariant derivative and the metric, respectively, of the ambient space. Let $W$ be a vector field along $\Sigma_{p}$ defined by $W=\lambda^{\prime} N /(\lambda-\mu)+\lambda T$. Then, $g_{\varepsilon}\left(W, V_{i}\right)=0$ and

$$
\bar{\nabla}_{V_{i}} W=\frac{\lambda^{\prime}}{\lambda-\mu} \bar{\nabla}_{V_{i}} N+\lambda \bar{\nabla}_{V_{i}} T=0 .
$$

Thus $\nabla_{V_{i}} W=\alpha x$, where

$$
\alpha=\varepsilon g_{\varepsilon}\left(\nabla_{V_{i}} W, x\right)=-\varepsilon g_{\varepsilon}\left(W, \nabla_{V_{i}} x\right)=-\varepsilon g_{\varepsilon}\left(W, V_{i}\right)=0
$$

It follows that $W$ is constant along $\Sigma_{p}$ and orthogonal to $\bar{P}^{n}$.

Now let $A=-x+N / \lambda$. Then $g_{\varepsilon}\left(A, V_{i}\right)=0$ and

$$
\nabla_{V_{i}} A=-V_{i}+\frac{1}{\lambda} \nabla_{V_{i}} N=-V_{i}+\frac{1}{\lambda} \bar{\nabla}_{V_{i}} N=0,
$$

which means that $A$ is also constant along $\Sigma_{p}$ and orthogonal to $\bar{P}^{n}$. Since $W$ and $A$ are linearly independent, we can consider the plane $\Pi=[W, A]$ generated by $W$ and $A$. $\Pi$ is the subspace orthogonal to $\bar{P}^{n}$, and to prove that $\bar{P}^{n}$ is parallel it suffices to show that $\Pi$ is parallel. Thus we must show that both $\nabla_{T} W$ and $\nabla_{T} A$ belong to $\Pi$.

Since the ambient space is flat and $\left[T, V_{i}\right]=0$, we obtain

$$
\nabla_{V_{i}} \nabla_{T} A=\nabla_{T} \nabla_{V_{i}} A=0, \quad \nabla_{V_{i}} \nabla_{T} W=\nabla_{T} \nabla_{V_{i}} W=0 .
$$

Thus $\nabla_{T} A$ and $\nabla_{T} W$ are constant along $\Sigma_{p}$. Furthermore,

$$
g_{\varepsilon}\left(\nabla_{T} A, V_{i}\right)=-g_{\varepsilon}\left(A, \nabla_{T} V_{i}\right)=-g_{\varepsilon}\left(A, \bar{\nabla}_{T} V_{i}\right)=-g_{\varepsilon}\left(A, \bar{\nabla}_{V_{i}} T\right)=0
$$


and

$$
g_{\varepsilon}\left(\nabla_{T} W, V_{i}\right)=-g_{\varepsilon}\left(W, \bar{\nabla}_{V_{i}} T\right)=0 .
$$

It follows that both $\nabla_{T} W$ and $\nabla_{T} A$ are constant vectors along $\Sigma_{p}$ that are orthogonal to $\bar{P}^{n}$, hence belong to $\Pi$, as we wished to prove.

Notice that if the subspace $P^{n}$ parallel to $\bar{P}^{n}$ is degenerate, the same happens with $\Pi$ and furthermore $\operatorname{dim}\left(P^{n} \cap \Pi\right)=1$; however, it is still true that the parallelism of $\Pi$ implies that of $\bar{P}^{n}$.

Now assume that $\bar{M}^{n+1}(c)$ is not $R^{n+1}$. Choose a basis $e_{1}, \ldots, e_{n+2}$ of the ambient space such that:

(i) If the subspace parallel to $\bar{P}^{n}$ is either Riemannian or Lorentzian $e_{1}, \ldots, e_{n+2}$ is an orthonormal basis with $g_{\varepsilon}\left(e_{1}, e_{1}\right)=\varepsilon$, in the first case, and $g_{-1}\left(e_{n+2}, e_{n+2}\right)=-1$, in the second case. In both cases, the basis is so chosen that $\bar{P}^{n}$ is parallel to $\left[e_{1}, \ldots, e_{n}\right]$. We will denote $\left[e_{n+1}, e_{n+2}\right]=P^{2}$.

(ii) If the subspace parallel to $\bar{P}^{n}$ is degenerate, $e_{1}, \ldots, e_{n+2}$ is a pseudo-orthonormal basis with $g_{-1}\left(e_{1}, e_{1}\right)=g_{-1}\left(e_{n+1}, e_{n+1}\right)=0, g_{-1}\left(e_{1}, e_{n+1}\right)=1$, and chosen in a way that $\bar{P}^{n}$ is parallel to $\left[e_{2}, \ldots, e_{n+1}\right]$. We will denote $\left[e_{n+1}, e_{n+2}\right]=P^{2}$.

In any case, as $\bar{P}^{n}$ moves along the leaf $L_{p}$, its intersection with $\bar{M}^{n+1}(c)$ describes a rotation hypersurface around $P^{2}$ that is exactly $f\left(M^{n}\right)$ as we wished to prove.

In case $\bar{M}^{n+1}(c)=R^{n+1}$, the above argument does not work, and we must show that the $(n-1)$-spheres $\Sigma_{p}$ that are in parallel $n$-planes $\bar{P}^{n}$ have their centers lying on a straight line orthogonal to $\bar{P}^{n}$.

For that, we define a map $Y: M^{n} \rightarrow R^{n+1}$ by

$$
Y(q, t)=f(q, t)-N / \lambda(t), \quad q \in \Sigma_{p}, t \in L .
$$

It is easily seen that $\bar{\nabla}_{V_{i}} Y=0$; thus $Y$ is a function of $t$ alone, i.e., $Y=Y(t)$. Notice that the sphere with center $Y(t)$ and radius $1 / \lambda(t)$ is tangent to $M$ along $\Sigma$. Thus we are reduced to showing that $Y(t)$ is a straight line orthogonal to $\bar{P}^{n}$. Since

$$
\bar{\nabla}_{T} Y=T-\frac{1}{\lambda} \bar{\nabla}_{T} N+\frac{\lambda^{\prime}}{\lambda^{2}} N=\frac{\lambda-\mu}{\lambda} T+\frac{\lambda^{\prime}}{\lambda^{2}} N,
$$

we have that $g_{1}\left(\bar{\nabla}_{T} Y, V_{i}\right)=0$. Thus

$$
g_{i}\left(\bar{\nabla}_{T} \bar{\nabla}_{T} Y, V_{i}\right)=-g_{1}\left(\bar{\nabla}_{T} Y, \bar{\nabla}_{T} V_{i}\right)=0 .
$$

It follows that both $\bar{\nabla}_{T} Y$ and $\bar{\nabla}_{T} \bar{\nabla}_{T} Y$ are orthogonal to $\bar{P}^{n}$. Therefore $\bar{\nabla}_{T} \bar{\nabla}_{T} Y$ is either zero or parallel to $\bar{\nabla}_{T} Y$. In both cases, $Y(t)$ describes a straight line orthogonal to $\bar{P}^{n}$, and this shows that, in a neighborhood of $p, f$ is a rotation hypersurface.

Now, from Corollary (3.8), we see that a rotation hypersurface is locally determined by the functions $\lambda(s)$ and $\mu(s)$. By a standard connectedness argument, we conclude that $f(M)$ is contained in a rotation hypersurface, as we wished to prove.

(4.3) REMARK. Theorem (4.2) is clearly false for $n=2$.

For the case $c>0$, the following corollary has been obtained by Otsuki [10].

(4.4) COROLlaRY. Let $f: M^{n} \rightarrow \bar{M}^{n+1}(c), n \geqslant 3$, be a minimal hypersurface that has two principal curvatures $\lambda$ and $\mu$, where one of them, say $\lambda$, has multiplicity at least $n-1$. Then $f(M)$ is contained in a catenoid. 
Proof. By minimality, $f$ is analytic. If there exists a neighborhood of umbilic points, $f$ is totally geodesic and the corollary holds. Otherwise, there exists a neighborhood of nonumbilic points and Theorem (4.2) applies to this neighborhood. By analyticity, $f(M)$ is contained in a catenoid. Q.E.D.

It is a known fact (see e.g. [9]) that for $n \geqslant 4$ a hypersurface $f: M^{n} \rightarrow \bar{M}^{n+1}(c)$ is conformally flat if and only if at least $n-1$ of its principal curvatures are equal, say, to $-\lambda$. Let the other principal curvature be denoted by $-\mu$. The following is an immediate consequence of Theorem (4.2).

(4.5) COROLlaRY. Let $f: M^{n} \rightarrow \bar{M}^{n+1}(c), n \geqslant 4$, be a conformally flat hypersurface. Assume that $\lambda \neq 0, \mu=\mu(\lambda)$ and $\lambda-\mu \neq 0$. Then $f(M)$ is contained in a rotation hypersurface.

For the case $c=0$, the following corollary has been obtained by Blair [2].

(4.6) Corollary. Let $f: M^{n} \rightarrow \bar{M}^{n+1}(c), n \geqslant 4$, be a conformally flat minimal hypersurface. Then $f(M)$ is contained in a catenoid.

The proof is the same as in Corollary (4.4).

The next theorem is related to the question of determining which hypersurfaces of $\bar{M}^{n+1}(c)$ are invariant by an $l$-parameter subgroup of isometries of $\bar{M}^{n+1}(c)$ and have a specified property. Roughly speaking, we show that if we exclude the hypersurfaces that have constant principal curvatures and assume that $l$ is the maximum admissable value, then $f(M)$ is a rotation hypersurface.

It will be convenient to denote by $\mathcal{C}(n, c)$ the set of hypersurfaces of $\bar{M}^{n+1}(c)$ that have constant principal curvatures.

(4.7) THEOREM. Let $n \geqslant 3$ be fixed and let $f: M^{n} \rightarrow \bar{M}^{n+1}(c)$ be a hypersurface invariant by an l-parameter subgroup of the isometries of $\bar{M}^{n+1}(c)$. Assume that $f \notin \circlearrowright$. Then the maximum value of $l$ is $l_{\max }=n(n-1) / 2$, and if $l=l_{\max }$, f has two principal curvatures $\lambda$, $\mu$, where one of them, say $\lambda$, has multiplicity at least $n-1$. If, in addition, $\lambda \neq 0, \mu=\mu(\lambda)$ and $\mu-\lambda \neq 0$, then $f(M)$ is a rotation hypersurface.

Proof. The last sentence is an immediate consequence of Theorem (4.2).

To prove the first part of Theorem (4.7), we begin by showing that $l_{\max }=$ $n(n-1) / 2$. That this value can be reached follows from the existence of rotation hypersurfaces. Let $p \in M^{n}$ and let $\Sigma_{p}$ be the orbit of $p$ under the orbit of an $l_{\max }$-parameter subgroup of the isometries of $\bar{M}^{n+1}(c)$. Assume that $l_{\max }>$ $n(n-1) / 2$. Then $\operatorname{dim} \Sigma_{p}=n$; otherwise the dimension of the group of isometries of $\Sigma_{p}$ is at most $n(n-1) / 2$. Since $\operatorname{dim} \Sigma_{p}=n$, by homogeneity, $\Sigma_{p}=M^{n}$. But then $M^{n} \in \mathcal{C}$. This contradicts the hypothesis and shows that $l_{\max }=n(n-1) / 2$.

Now let $l=l_{\max }=n(n-1) / 2$. We want to show that $f$ has two principal curvatures and one of them has at least multiplicity $n-1$. Let again $p \in M^{n}$ and $\Sigma_{p}$ be the orbit of $p$ under the action of the l-parameter subgroup. Since $f \notin e$, $\operatorname{dim} \Sigma_{p}=n-1$. If $p$ is umbilic, the theorem holds; thus we can assume that $p$ is not an umbilic.

Let $e_{1}, \ldots, e_{n}$ be unit principal vectors at $p$ with principal curvatures $k_{1}, \ldots, k_{n}$, respectively. We first show that the tangent space $T_{p}\left(\Sigma_{p}\right)$ of $\Sigma_{p}$ at $p$ contains $n-1$ 
principal vectors. This amounts to proving that if a unit vector $v$ belongs to $T_{p}\left(\Sigma_{p}\right) \subset T_{p}(M)$, i.e., if $v=\sum \alpha_{i} e_{i}, \Sigma \alpha_{i}^{2}=1, \Sigma A_{i} \alpha_{i}=0, A_{i}$ constants, $i=1, \ldots, n$, then some $\alpha_{i}=0$.

Since the group acting on $\Sigma_{p}$ is a maximal group of isometries, and $n>2$, all normal curvatures in the directions contained in $T_{p}\left(\Sigma_{p}\right)$ are equal. This means that the function

$$
F\left(\alpha_{1}, \ldots, \alpha_{n}\right)=\sum k_{i} \alpha_{i}^{2}=\text { normal curvature in }\left(\alpha_{1}, \ldots, \alpha_{n}\right)
$$

restricted to the submanifold of $T_{p}\left(\Sigma_{p}\right)$ defined by

$$
\left\{\begin{array}{l}
G\left(\alpha_{1}, \ldots, \alpha_{n}\right)=\sum \alpha_{i}^{2}-1=0, \\
H\left(\alpha_{1}, \ldots, \alpha_{n}\right)=\sum A_{i} \alpha_{i}=0,
\end{array}\right.
$$

is constant. By using the method of Lagrange multipliers we obtain that, for some real numbers $\lambda$ and $\beta, \nabla(F+\lambda G+\beta H)=0$, where $\nabla()$ denotes the gradient of the enclosed function. Thus

$$
2 \alpha_{i}\left(k_{i}+\lambda\right)+\beta A_{i}=0, \quad i=1, \ldots, n,
$$

for all $\alpha_{i}$. If $\beta \neq 0$, then $A_{i}=0$ for all $i$, and this is impossible. Thus $\beta=0$ and $\alpha_{i}\left(k_{i}+\lambda\right)=0$. If all $\alpha_{i} \neq 0$, then all $k_{i}$ are equal and this contradicts the fact that $p$ is nonumbilic. Thus some $\alpha_{i}=0$ and this proves our claim. Notice that the proof does not work if $n=2$.

It follows that $(n-1)$ of the principal curvatures, say, $k_{1}, \ldots, k_{n-1}$ are equal, $k_{1}=\cdots=k_{n-1}=-\lambda$, and this completes the proof.

(4.8) Remark. The class $\bigodot(n, c)$ is the class of isoparametric families of $\bar{M}^{n+1}(c)$ in the sense of Elie Cartan. For the case $c \leqslant 0$ they have been classified (Cartan [4]) and there are not too many of them. Thus the restriction $f \notin \mathcal{C}(n, c), c \leqslant 0$, is not really serious. However, for $c>0$, the complete description of the class $\mathcal{C}(n, c)$ is still an open problem (see Ferus [6] for recent developments). Of course, not all elements of $\mathcal{C}(n, c), c>0$, are invariant by an $l$-parameter subgroup of the group of isometries of $\bar{M}^{n+1}(c)$. If the classification could be obtained under this additional restriction, Theorem (4.7) could be made more explicit.

In any case, for minimal hypersurfaces we can be more precise.

(4.9) Corollary. Let $f: M^{n} \rightarrow \bar{M}^{n+1}(c), c \leqslant 0, n \geqslant 3$, be a minimal hypersurface, not totally geodesic, invariant by an l-parameter subgroup of the isometries of $\bar{M}^{n+1}(c)$. Then $l_{\max }=n(n-1) / 2$ and if $l=l_{\max }, f(M)$ is a catenoid (i.e., a rotation minimal hypersurface).

This follows from Theorem (4.7), the fact that the only minimal hypersurface in $\bigodot(n, c), c \leqslant 0$, is totally geodesic, and the argument given in Corollary (4.4).

(4.10) Remark. For the case that the ambient space is $S^{n+1}(c), n \geqslant 3$, the following examples show that Corollary (4.9) does not hold. The Clifford minimal hypersurfaces $S^{n-1} \times S^{1} \subset S^{n+1}(1)$ are catenoids invariant by an $((n(n-1) / 2)+$ 1)-parameter group of isometries of $S^{n+1}(1)$, and the Clifford minimal hypersurface $S^{2} \times S^{2} \subset S^{5}(1)$ is invariant by an $(n(n-1) / 2=6)$-parameter group of isometries 
of $S^{5}(1)$ but is not a catenoid. From the above proof, it is easy to see that if $l=n(n-1) / 2$ and $\operatorname{dim} \Sigma_{p}=n-1$, where $\Sigma_{p}$ is the orbit of $p \in M$, then $f(M)$ is a nonhomogeneous catenoid.

\section{Stability of catenoids.}

(5.1) In this section we will describe some stability properties of the rotation minimal surfaces in hyperbolic space $H^{n+1}(c)$; for computational convenience, we state the results for $c=-1$.

We start with $n=2$. In this case, the catenoids in $H^{3}(-1)$ are parametrized by (see (3.20))

$$
x_{1}(s)=(a \cosh 2 s-\delta / 2)^{1 / 2},
$$

where $a>1 / 2$ if $\delta \neq 0$ and $a=1$ if $\delta=0$. Furthermore, the square of the norm $|A|^{2}$ of the second fundamental form $A$ is given by

$$
|A|^{2}=\frac{2\left(a^{2}-|\delta| / 4\right)}{(a \cosh 2 s-\delta / 2)^{2}},
$$

and satisfies

$$
\begin{gathered}
|A|^{2} \leqslant \frac{2\left(a^{2}-1 / 4\right)}{(a-\delta / 2)^{2}}=\frac{2(a+\delta / 2)}{(a-\delta / 2)}<2 \text { if } \delta=-1, \\
|A|^{2} \leqslant \frac{2}{\cosh ^{2} 2 s} \leqslant 2 \text { if } \delta=0 .
\end{gathered}
$$

It has been proved by $\mathrm{H}$. Mori [8] that, if $a>17 / 2$, spherical catenoids are globally stable. This follows from the second variation formula for a domain $D \subset M$,

$$
\begin{aligned}
I_{D}(u N, u N) & =-\int_{D}\left\{u \Delta u+\left(|A|^{2}-2\right) u^{2}\right\} d M \\
& \geqslant \int_{D}\left(\lambda_{1}(D)+2-|A|^{2}\right) u^{2} d M
\end{aligned}
$$

here $N$ is a unit normal vector, $\Delta u$ is the Laplacian of the function $u(u=0$ on $\partial D)$ in the induced metric and $\lambda_{1}(D)$ is the first eigenvalue of the Laplacian in the induced metric. By observing that $|A|^{2} \leqslant 2(a+1 / 2) /(a-1 / 2)$ and that, for every domain $D$ of a negatively curved surface homeomorphic to a cylinder, $\lambda_{1}(D)>1 / 4$, we conclude that, for $a>17 / 2, I_{D}(u N, u N)>0$, for all $D$ and all $u$ with $u=0$ on $\partial D$. Thus spherical catenoids are globally stable if $a>17 / 2$. Notice that spherical catenoids are not simply-connected.

Our computations (5.2) and (5.3) for the hyperbolic and parabolic catenoids, together with (5.4), allow us to make the following assertion.

(5.5) THEOREM. Hyperbolic and parabolic catenoids in $H^{3}(-1)$ are globally stable. Thus there exist a family of simply-connected, complete, embedded nontotally geodesic stable minimal surfaces in $H^{3}(-1)$.

The last sentence follows from the first one and Theorem (3.26). 
The question now arises whether there exists some unstable catenoid in $H^{3}(-1)$. Our answer to this question will depend on a general theorem that may be interesting in its own right and can be stated as follows.

(5.6) THEOREM. Let $f: M^{n} \rightarrow \bar{M}^{n+1}(c)$ be a complete, stable minimal immersion. Let $S$ be the scalar curvature of $M$ in the induced metric, denote by $B_{R}$ the geodesic ball of $M$ with a fixed center $p_{0} \in M$ and radius $R$, and assume that

$$
\lim _{R \rightarrow \infty} \frac{\int_{B_{R}}|(S-n(n-1) c)| d M}{R^{2+2 q}}=0, \quad 0<q<\sqrt{2 / n} .
$$

Then $c \leqslant 0$. If $c=0, f(M) \subset R^{n+1}$ is a hyperplane, and if $c<0$,

$$
\underset{R \rightarrow \infty}{\limsup } \int_{B_{R}}|A|^{2+2 q}\left(|A|^{2}+\beta\right) d M \leqslant 0,
$$

where $|A|^{2}=|S-n(n-1) c|$ is the square of the norm of the second fundamental form $A$ of $f$ and $\beta$ is a constant depending on $n, c$ and $q$ that will be defined during the proof.

Proof. Theorem (5.6) is a generalization of Theorem (1.3) in M. do Carmo and C. $\mathrm{K}$. Peng [3] and the proof follows essentially the same steps. We have only to replace (2.5) and (2.6) of [3] by

$$
\begin{gathered}
|A| \Delta|A|+|A|^{4} \geqslant \frac{2}{n}|\nabla| A||^{2}+n c|A|^{2}, \\
\int_{M}\left(n c+|A|^{2}\right) f^{2} d M \leqslant \int_{M}|\nabla f|^{2} d M .
\end{gathered}
$$

From that point on, we follow the proof of [3] up to (2.11) that is now replaced by

$$
\int_{M}|A|^{4+2 q} f^{2} d M+\beta \int_{M}|A|^{2 q+2} f^{2} d M \leqslant \beta_{1} \int_{M}|A|^{2 q+2}|\nabla f|^{2} d M,
$$

where $\beta_{1}$ is a constant depending on $n, c, q$ and $\beta$ is a similar constant given by

$$
\beta=n c\left[\frac{q^{2}+(4+\varepsilon) q+2+2 \varepsilon+2 / n}{2 / n-q^{2}-\varepsilon q}\right] ;
$$

here $\varepsilon>0$ is chosen so that

$$
\frac{1+q+\varepsilon}{q+2 / n} q<1 \quad \text { if } q<\sqrt{2 / n} .
$$

By using, as in [3], Young's inequality in (5.9), we obtain

$$
\int_{M}|A|^{2+2 q}\left(|A|^{2}+\beta\right) f^{2+2 q} d M \leqslant \beta_{2} \int_{M}|A|^{2}|\nabla f|^{2+2 q} d M,
$$


where again $\beta_{2}$ is a constant. From (5.11) we conclude as in [3] that

$$
\int_{B_{\theta R}}|A|^{2+2 q}\left(|A|^{2}+\beta\right) \leqslant \beta_{3} \frac{\int_{B_{R}}|A|^{2} d M}{(1-\theta)^{2+2 q} R^{2+2 q}}, \quad 1<\theta<1 .
$$

Now if we let $R \rightarrow \infty$ and use the hypothesis of the theorem, we obtain that the left-hand side of the above inequality is nonpositive. If $c>0$ then $\beta>0$, and this implies that $A=0$. Since there is no stable totally geodesic complete submanifold of a sphere, this is a contradiction and $c \leqslant 0$. If $c=0, \beta=0$ and we are reduced to the case proved in [3], namely $f(M)$ is a hyperplane of $R^{n+1}$. Finally, if $c<0$, we obtain that

$$
\limsup _{R \rightarrow \infty} \int_{B_{R}}|A|^{2+2 q}\left(|A|^{2}+\beta\right) d M \leqslant 0
$$

as we wished.

(5.13) COROllaRY. Let $f: M^{n} \rightarrow H^{n+1}(-1)$ be a complete minimal immersion with finite total curvature. If $f$ is globally stable then

$$
\int_{M}|A|^{2}\left(|A|^{2}-n(n+1) d M\right) \leqslant 0 .
$$

Proof. Notice that if we take $\varepsilon=0, q=0$, then from (5.10),

$$
\beta=-(2 n+2) /(2 n-1) \text {. }
$$

Since the integrals we are dealing with are finite, the conclusion follows. Q.E.D.

The answer to our question is an application of the above corollary. We first observe that spherical catenoids have finite total curvature. Indeed

$$
|A|^{2}=\frac{2\left(a^{2}-1 / 4\right)}{(a \cosh 2 s-1 / 2)^{2}}, \quad d M=(a \cosh 2 s-1 / 2)^{1 / 2} d s d t, \quad 0 \leqslant t \leqslant 2 \pi .
$$

Thus

$$
\begin{aligned}
\int_{M}|A|^{2} d M & =4 \pi\left(a^{2}-1 / 4\right) \int_{0}^{\infty} \frac{1}{(a \cosh 2 s-1 / 2)^{3 / 2}} d s \\
& <4 \pi\left(a^{2}-1 / 4\right) \int_{0}^{\infty} \frac{d s}{\left(a+a s^{2}-1 / 2\right)^{3 / 2}}<\infty .
\end{aligned}
$$

By Corollary (5.13), we see that should a spherical catenoid corresponding to the parameter $a$ be globally stable, then

$$
F(a)=\int_{0}^{\infty}\left(\frac{a^{2}-1 / 4}{(a \cosh 2 s-1 / 2)^{7 / 2}}-\frac{3}{(a \cosh 2 s-1 / 2)^{3 / 2}}\right) d s \leqslant 0 .
$$

Now, it is possible to show that if $1 / 2<a<c_{0}, c_{0} \simeq 0.69$, then $F(a)>0 .{ }^{1}$ Thus, such spherical catenoids are not globally stable.

\footnotetext{
${ }^{1}$ The above value was communicated to us by $\mathrm{H}$. Mori. A better value can probably be obtained by using a computer.
} 


\section{REFERENCES}

1. J. L. Barbosa and M. do Carmo, Helicoids, catenoids, and minimal hypersurfaces of $R^{n}$ invariant by an l-parameter group of motions, An. Acad. Brasil. Ciênc. 53 (1981), 403-408.

2. D. Blair, A generalization of the catenoid, Canad. J. Math. 27 (1975), 231-236.

3. M. do Carmo and C. K. Peng, Stable complete minimal hypersurfaces, Proc. Beijing Sympos. (to appear).

4. E. Cartan, Familles de surfaces isoparamétriques dans les espaces à courbure constant, Ann. Mat. Pura Appl. 17 (1938), 177-191.

5. C. Delaunay, Sur les surfaces de revolution dont la courbure moyenne est constante, J. Math. Pures Appl. (1) 6 (1841), 309-320.

6. D. Ferus, Notes on isoparametric hypersurfaces, Escola de Geometria Diferencial, Universidade de Campinas, São Paulo, Brasil, 1980.

7. H. B. Lawson, Jr., Complete minimal surfaces in $S^{3}$, Ann. of Math. (2) 92 (1970), 335-374.

8. H. Mori, Minimal surfaces of revolution in $H^{3}$ and their stability properties, Indiana Math. J. 30 (1981), 787-794.

9. S. Nishikawa and Y. Maeda, Conformally flat hypersurfaces in a conformally flat Riemannian manifold, Tôhoku Math. J. 26 (1974), 159-168.

10. T. Otsuki, Minimal hypersurfaces in a Riemannian manifold of constant curvature, Amer. J. Math. 92 (1970), 145-173.

11. P. Ryan, Homogeneity and curvature conditions for hypersurfaces, Tôhoku Math. J. 21 (1969), $363-388$.

Instituto de Matemática Pura e Aplicada (IMPA), Estrada D. Castorina, 110, Jardim Botânico, 22460, RIO DE JANEIRO, RJ-BRASIL 